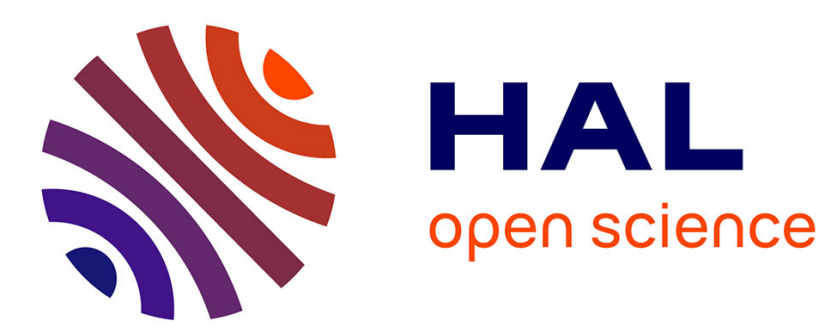

\title{
Direct measurements of the current-phase relation in superconducting weak links
}

\author{
J.R. Waldram, J.M. Lumley
}

\section{To cite this version:}

J.R. Waldram, J.M. Lumley. Direct measurements of the current-phase relation in superconducting weak links. Revue de Physique Appliquée, 1975, 10 (1), pp.7-10. 10.1051/rphysap:019750010010700 . jpa-00243878

\section{HAL Id: jpa-00243878 https://hal.science/jpa-00243878}

Submitted on 1 Jan 1975

HAL is a multi-disciplinary open access archive for the deposit and dissemination of scientific research documents, whether they are published or not. The documents may come from teaching and research institutions in France or abroad, or from public or private research centers.
L'archive ouverte pluridisciplinaire HAL, est destinée au dépôt et à la diffusion de documents scientifiques de niveau recherche, publiés ou non, émanant des établissements d'enseignement et de recherche français ou étrangers, des laboratoires publics ou privés. 


\title{
Classification \\ Physics Abstracts 8.440 \\ DIRECT MEASUREMENTS OF THE CURRENT-PHASE RELATION IN SUPERCONDUCTING WEAK LINKS
}

\author{
J. R. WALDRAM et J. M. LUMLEY \\ Physics Department, University of Birmingham, Birmingham England
}

(Reçu le 6 mai 1974)

\begin{abstract}
Résumé. - Une nouvelle technique est présentée qui rend possible la détermination à la fois directe et précise de la relation courant-phase dans un nombre de weak links supraconducteurs. Des résultats expérimentaux sont donnés ; quoique le comportement des junctions SNS utilisées soit dans une certaine mesure celui de jonction de grande surface il ne diffère pas d'une manière très significative de celui prédit par la relation de Josephson.
\end{abstract}

\begin{abstract}
A new type of experiment is reported in which an accurate direct measurement of the current-phase relation in a variety of superconducting weak links is possible. Results are presented for SNS junctions which, although showing some interesting large junction behaviour, show no substantial departure from the Josephson relation.
\end{abstract}

1. Introduction. - It is well known that a superconductor is characterised by an order parameter [1], $\Psi$, which behaves in some respects like a one-particle wave function having a well defined phase, $\theta$. In particular, in the bulk, the supercurrent is proportional to the superfluid momentum $m \mathbf{v}_{\mathrm{s}}=\lambda \nabla \theta+2 e \mathbf{A}$. But it is also well known that, for a number of different cases where pairs of superconductors are weakly coupled together in some way, the supercurrent which flows across the weak link is not linear but periodic in the phase difference across the link ; indeed many cases exhibit simple Josephson behaviour [2, 3, 4]

$$
i_{\mathrm{s}}=i_{\mathrm{J}} \sin \varphi
$$

where $\varphi$ is the gauge invariant phase difference across the link,

$$
\varphi=\theta_{2}-\theta_{1}+\frac{2 e}{\hbar} \int_{1}^{2} \mathbf{A} \cdot \mathbf{d l} .
$$

This behaviour was first predicted by Josephson [2] for tunnel junctions (SIS junctions); the same result has been obtained for superconducting-normal-superconducting junctions (SNS) by de Gennes [5], and for sufficiently small microbridges by Baratoff et al. [6] using Ginzburg-Landau theory. Experimental confirmation, however, has usually been somewhat indirect and of relatively low precision ; the best confirmation comes from the interference patterns seen in large junctions [7, 8], or circuits containing double junctions in an applied magnetic field $[9,10]$, and from the successful prediction of the high frequency effects which follow from (1) [11].

The aim of the experiment reported here is to make a direct measurement in static conditions of the current-phase relation for a wide range of weak links, to a precision better than $1 \%$. In particular, we wished to look at such cases as long SIS and SNS junctions, long or wide microbridges, microbridges at temperatures well below $T_{\mathrm{c}}$, and $\mathrm{SS}^{\prime} \mathrm{S}$ junctions at temperatures above $T_{\mathrm{c}}^{\prime}$ for $\mathrm{S}^{\prime}$ where experiment or theory suggest that there may be departures from the Josephson relation [6, 12]. However, in this letter we simply describe the method adopted and limited results for small SNS junctions; we shall report on other work at a later date.

2. Method. - The principle of the measurement is straightforward. The weak link is inserted into a superconducting loop as shown in figure $1 a$. Quite generally, the phase change measured around the loop must be the same as that across the junction. Because supercurrents are screened from a bulk superconductor, the superfluid momentum is zero along path $C$, and integration around the loop yields for the phase difference, $\theta_{2}-\theta_{1}$,

$$
\theta_{2}-\theta_{1}=\frac{2 e}{\hbar} \int_{2 \text { along c }}^{1} \text { A.dl . }
$$

Using this value in eq. $(1 b)$ yields a simple result of great importance,

$$
\begin{aligned}
\varphi & =\frac{2 e}{\hbar} \oint \mathbf{A} \cdot \mathbf{d l} \\
& =2 \pi \frac{\Phi}{\Phi_{0}}
\end{aligned}
$$


(a)

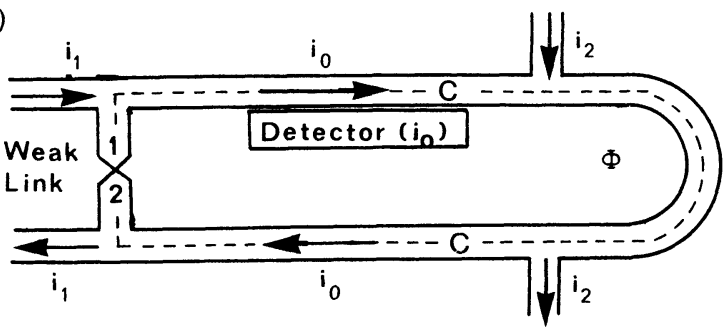

(b)

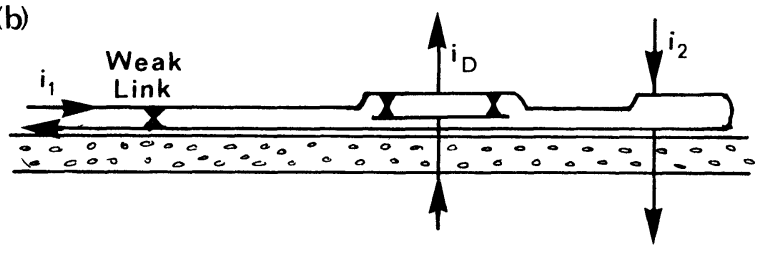

(c)

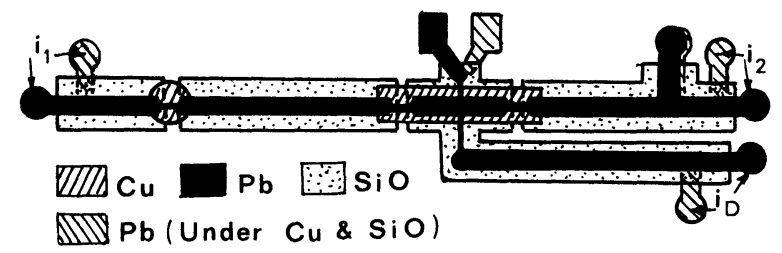

FIG. 1. - Schematic and Experimental Circuity. a) Schematic. b) Section through evaporated circuit. c) Plan view-indicating the manner in which the circuit is formed.

where $\Phi_{0}$ is the flux quantum, $h / 2 e$. Eq. (3) is of complete generality and implies that a current $j_{\mathrm{c}} \sin 2 \pi \Phi / \Phi_{0}$ flows through the weak link when the total flux in the loop is $\Phi$.

This result suggests the following procedure as illustrated in figure $1 a$. Also inserted into the superconducting loop is a detector which is sensitive to the passage of the current $i_{0}$ only.

(i) Insert $i_{1}$ : This current will divide so that equal phases are produced around the loop and across the junction. The detector responds to the circulating current, $i_{0}$.

(ii) Insert $i_{2}$ : Similarly, this current will divide and if inserted with the correct polarity, the value of $i_{0}$ will be reduced.

(iii) When $i_{0}$ is zeroed, the circuit is said to be balanced; in this condition, $i_{1}$ flows through the junction and $i_{2}$ flows around the end of the loop. From figure $1 b$, it is also clear that the total flux in the loop is then proportional to $i_{2}$.

(iv) Hence, from eq. (3), it can be seen that a plot of $i_{1}$ vs. $i_{2}$ at balance will yield the current-phase relation.

The difficulty in practice is to determine the flux, $\Phi$, with the required precision while simultaneously inserting a current detector of the desired sensitivity into the loop. The experimental arrangement adopted was to prepare the circuit by vacuum evaporation into a microscope slide, as shown in figures $1 b, c$.

2.1 We NOTE THE FOLLOWING DETAILS. - (i) When the current, $i_{0}$ is zeroed, the link current, $i_{1}$, is folded back on itself producing very little stray field. The thickness of the insulating layer inside the main loop is typically $\sim 10^{-7} \mathrm{~m}$, and its cross sectional area $\sim 3 \times 10^{-9} \mathrm{~m}^{2}$ implying a self inductance $\sim 10^{-11} \mathrm{H}$. Calculations show that the flux fed back into the main loop by a link current $\sim 1 \mathrm{~mA}$ should be less than $\sim 2 \times 10^{-4}$ flux quanta, and there is some internal evidence to confirm this. Mu-metal cans and a superconducting shield were used to stabilise stray flux at $<10^{-1}$ flux quanta.

(ii) The null meter consists of a double SNS junction inserted into the main loop. Its critical current, $i_{\mathrm{c}}$, is a periodic function of the flux induced by the circulating current, $i_{0}$, in the enclosed area of the double junction. Variations in $i_{\mathrm{c}}$ are detected by biasing the current, $i_{\mathrm{D}}$, through the double junction at a fixed value just in excess of $i_{\mathrm{c}}$, and then observing the changes in voltage appearing across the double junction with a slug circuit [13], these voltages being $\sim 10^{-11} \mathrm{~V}$. At optimum sensitivity, about $10^{-3}$ flux quanta can be detected by the double junction, corresponding to a change in $i_{0}$ of $\sim 0.1 \mu \mathrm{A}$ and a simultaneous change in $\Phi$ of about $2 \times 10^{-3}$ flux quanta (the area of the double junction loop is about half the total area of the main loop).

(iii) It is not necessary either to calibrate the flux induced by $i_{2}$ in advance, or that $i_{0}$ should be zeroed. Nor does it matter if stray fields or asymmetric currents in the double junction inject flux into the main loop. The flux is self calibrating, because of the periodic nature of the $i-\varphi$ relation, and a finite constant value of $i_{0}$ simply shifts the origin of the $i_{1}-i_{2}$ plot, as seen in the results of figure $2 a$. Thus, since the measurements are always made with the double junction in the same state, the magnetic effects of $i_{0}$ and $i_{\mathrm{D}}$ are unimportant.

It is true that $i_{0}$ will be affected by magnetic interaction from either $i_{1}$ or $i_{2}$. Such magnetic feedback is undesirable since it causes a distortion of the $i_{1}-i_{2}$ plot and is hard to differentiate from a genuine departure from the Josephson relation. For the results to be presented, this effect was felt to be small.

(iv) If the critical current is adjusted so that the inductance, $L$, is less than $\Phi_{0} / 2 \pi i_{\mathrm{J}}$, then flux jumps in the main loop do not occur [9]. Then the whole of the current-phase curve can be plotted out, including those regions for which $\partial i / \partial \varphi$ is negative, which are unstable when the weak link is simply connected to a source of current. The above condition is met for $i_{\mathrm{J}}<10 \mu \mathrm{A}$ in the experiments.

(v) Although the above analysis used an integration path deep within the superconductor, and although in the experiments the film thicknesses were rather 

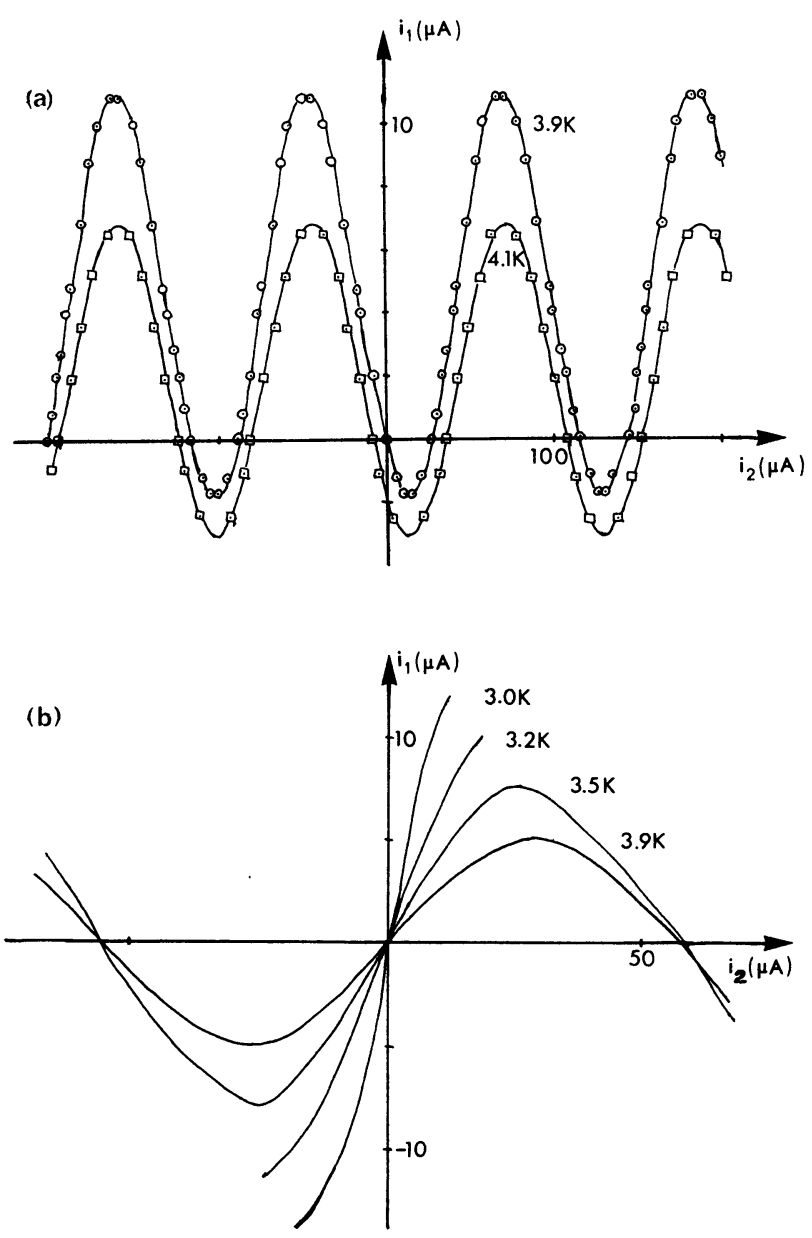

Fig. 2. - Junction " $A$ » results. The temperatures indicated are approximate. a) Current-phase results over many periods of phase. The points are experimental and the solid lines are sine waves of appropriate amplitude and periodicity. The agreement is very good indeed. $b$ ) Results down to lower temperatures. Notice that it is impossible to trace out the whole curve for critical currents $\gtrsim 10 \mu \mathrm{A}$. These curves are experimental.

greater than the magnetic penetration depth, these restrictions are not essential. If the current density, $\mathbf{J}_{\mathrm{s}}$, is non zero along the integration path, eq. (1b) then yields,

$$
\varphi=\frac{2 \pi}{\Phi_{0}}\left(\Phi+\int_{1 \text { along } \mathrm{c}}^{2} \Lambda \mathbf{J}_{\mathrm{s}} \cdot \mathrm{dl}\right)
$$

the expression in brackets being analogous to London's Fluxoid [14]. Since the fluxoid is also clearly proportional to $i_{2}$, the experimental current-phase relation is not affected in form. In this connection, one must remember that the effective magnetic areas of the loops include the penetration depths, and indeed, measurements of the period of $i_{2}$ provide an estimate of the temperature dependence of the penetration depth in the superconducting films used.

3. Results. - As an illustration of typical results we show, in figures 2,3 the $i_{1}-i_{2}$ plots for two $\mathrm{Pb}-\mathrm{Cu} / \mathrm{Al}$ $\mathrm{Pb}$ SNS junctions. Each junction was $0.1 \mathrm{~mm}$ wide and about $5 \times 10^{-7} \mathrm{~m}$ thick. Junction $A$ was $0.3 \mathrm{~mm}$ long whereas junction $B$ was $3 \mathrm{~mm}$ long.

The zero offsets due to the finite value of $i_{0}$ are obvious in figure $2 a$, which presents small critical current results for junction A. The smooth curves are not best fits to the results but are sine curves of appropriate amplitude and periodicity. The good agreement is self evident.

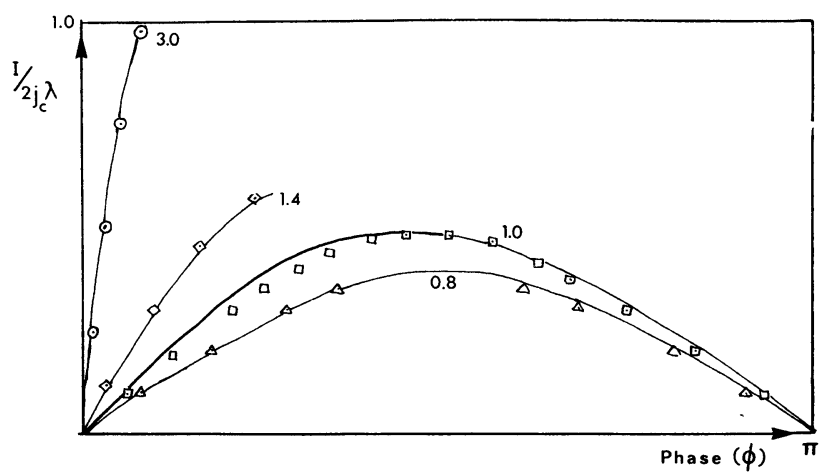

FIG. 3. - Junction «B $»$ results. The numbers indicated are the values of the ratio : $L / \lambda_{J}$ where $L$ is the junction length, $\lambda_{J}=\left(\hbar / 2 e \mu_{0} d j_{c}\right)^{1 / 2}$. is the Josephson penetration depth, and $d$ is the total magnetic thickness of the junction. The points are experimental and the solid lines are theoretical.

In figure $2 b$, it can be seen that the whole of the current-phase relation can be plotted out only for $i_{\mathrm{c}} \lesssim 10 \mathrm{~A}$, as predicted. Complete current-phase relations for larger critical currents could have been obtained only by reducing the inductance of the main loop. As the temperature was reduced, the critical current increased, and there appeared to be a departure from the Josephson relation.

To gain an idea of the nature of this departure, it must be realised that for finite length junctions, self fields affect the uniformity of the phase difference, $\varphi$, along the junction length, as per the results of Owen and Scalapino [15]. The experiment determines the phase at the end of the junction which faces into the loop and the current measured is the total current, which is injected at the other end of the junction.

Although beyond the scope of this paper, detailed calculations have been performed which, as for the calculations of Owen and Scalapino, give the variation with position of the phase difference in terms of elliptic integral solutions, but because the magnetic field was assumed to be zero, the present results do not allow flux quanta to exist within the junction. The solutions exhibit a Meissner effect and this was initially thought to be the reason for the apparent departure from the Josephson relation seen in figure $2 b$. However, detailed calculations showed that this was not the case. Also, magnetic feedback calculations were attempted which, although explaining some of the departures seen, could not explain them all. Consequently, for specimen $\mathrm{A}$, there really does seem to be a small departure from the Josephson relation.

The results obtained for junction $\mathbf{B}$ are presented 
in figure 3 together with a comparison with the theory mentioned above. It can be seen that there is really quite good agreement between experiment and theory. Notice that, in this figure, the vertical axis is a reduced current, $I / 2 j_{\mathrm{c}} \lambda_{\mathrm{J}}$, reflecting the result that the maximum current possible, irrespective of the junction size, is $2 j_{\mathrm{c}} \lambda_{\mathrm{J}}$.

It is clear that there is much to be learnt by applying measurements of this type to a large range of weak links, especially if, as is easily arranged, their currentvoltage characteristics and their high frequency response can be measured as well. The reason why there seems to be a departure for specimen A but not for specimen B is not clear at present and this would be the sort of result to be investigated in a more thorough study.

Acknowledgments. - We would both like to thank the members of the Low Temperature Physics workshop for their excellent technical assistance and one of us (JML) would like to express his gratitude to the Royal Society for the award of the Rutherford Scholarship during the period in which this research was carried out.

\section{References}

[1] Werthamer, N. R., Superconductivity (ed. Parks), (Marcell Dekker, Inc., New York) 1969, Vol. 1, p. 320.

[2] Josephon, B. D., Phys. Lett. 1 (1962) 251.

[3] Josephson, B. D., Superconductivity (Ed. Parks) Vol. 1, p. 423.

[4] Josephon, B. D., Adv. Phys. 14 (1965) 419.

[5] De Gennes, P. G., Phys. Lett. 5 (1963) 22.

[6] Baratoff, A., Blackburn, J. A., Schwartz, B. B., Phys. Rev. Lett. 25 (1970) 1096.

[7] Rowell, J. M., Phys. Rev. Lett., 11 (1963) 200.

[8] Matisoo, J., J. Appl. Phys., 40 (1969) 1813.
[9] Jaclevic, R. C., Lambe, J., Mercereau, J. E., Silver, A. H., Phys. Rev. A, 140 (1965) 1628.

[10] De Bruyn Ouboter, R., de Waele, A. Th. A. M., Progress in Low Temperature Physics (ed. Gorter) (North Holland, Amsterdam) 1970, Vol. VI, p. 243.

[11] Grimes, C. C., Shapiro, S., Phys. Rev. 169 (1968) 397.

[12] Bardeen, J., Johnson, J. L., Phys. Rev. B 5 (1972) 72.

[13] Clarke, J., Phil. Mag., 13 (1966) 115.

[14] London, F., Superfluids, (Chapman \& Hall Ltd. London) 1950, Vol. 1, p. 48

[15] Owen, C. S. et Scalapino, D. J., Phys. Rev. 164 (1967) 538. 